\title{
Energy Shift of H-Atom Electrons Due to Gibbons-Hawking Thermal Bath
}

\author{
Miroslav Pardy \\ Department of Physical Electronics, Laboratory of Plasma Physics, Masaryk University, Brno, Czech Republic \\ Email: pamir@physics.muni.cz
}

How to cite this paper: Pardy, M. (2016) Energy Shift of H-Atom Electrons Due to Gibbons-Hawking Thermal Bath. Journal of High Energy Physics, Gravitation and Cosmology, 2, 472-477.

http://dx.doi.org/10.4236/jhepgc.2016.24041

Received: May 18, 2016

Accepted: August 21, 2016

Published: August 24, 2016

Copyright $\odot 2016$ by author and Scientific Research Publishing Inc. This work is licensed under the Creative Commons Attribution International License (CC BY 4.0). http://creativecommons.org/licenses/by/4.0/

\begin{abstract}
The electromagnetic shift of energy levels of $\mathrm{H}$-atom electrons is determined by calculating an electron coupling to the Gibbons-Hawking ectromagnetic field thermal bath. Energy shift of electrons in $\mathrm{H}$-atom is determined in the framework of nonrelativistic quantum mechanics.
\end{abstract}

\section{Keywords}

H-Atom, Coulomb Potential, Blackbody Spektrum, Energy Shift

The Gibbons-Hawking effect is the statement that a temperature can be associated to each solution of the Einstein field equations that contains a causal horizon. It is named after Gary Gibbons and Stephen William Hawking.

Schwarzschild spacetime contains an event horizon and so can be associated with temperature. In the case of Schwarzschild spacetime this is the temperature $T$ of a blackhole of mass $M$, satisfying $T / M$.

De Sitter space which contains an event horizon has the temperature $T$ proportional to the Hubble parameter $H$. We consider here the influence of the heat bath of the Gibbons-Hawking photons on the energy shift of $\mathrm{H}$-atom electrons.

The considered problem is not in the scientific isolation, because some analogical problems are solved in the scientific respected journals. At present time it is a general conviction that there is an important analogy between black hole and the hydrogen atom. The similarity between black hole and the hydrogen atom was considered for instance by Corda [1], who discussed the precise model of Hawking radiation from the tunnelling mechanism. In this article an elegant expression of the probability of emission is given in terms of the black hole quantum levels. So, the system composed of Hawking radiation and black hole quasi-normal modes introduced by Corda [2] is 
somewhat similar to the semiclassical Bohr model of the structure of a hydrogen atom.

The time dependent Schrödinger equation was derived for the system composed by Hawking radiation and black hole quasi-normal modes [3]. In this model, the physical state and the correspondent wave function are written in terms of a unitary evolution matrix instead of a density matrix. Thus, the final state is a pure quantum state instead of a mixed one and it means that there is no information loss. Black hole can be well defined as the quantum mechanical systems, having ordered, discrete quantum spectra, which respect 't Hooft's assumption that Schrödinger equations can be used universally for all dynamics in the universe.

Thermal photons by Gibbons and Hawking form so called blackbody, which has the distribution law of photons derived in 1900 by Planck [4]-[6]. The derivation was based on the investigation of the statistics of the system of oscillators inside of the blackbody. Later Einstein [7] derived the Planck formula from the Bohr model of atom where electrons have the discrete energies and the energy of the emitted photons which are given by the Bohr formula $\hbar \omega=E_{i}-E_{f}$, where $E_{i}, E_{f}$ are the initial and final energies of electrons.

Now, let us calculate the modified Coulomb potential due to blackbody. The starting point of the determination of the energy shift in the $\mathrm{H}$-atom is the potential $V_{0}(x)$, which is generated by nucleus of the $\mathrm{H}$-atom. The potential at point $V_{0}(\boldsymbol{x}+\delta \boldsymbol{x})$, evidently is [8] [9]:

$$
V_{0}(\boldsymbol{x}+\delta \boldsymbol{x})=\left\{1+\delta \boldsymbol{x} \nabla+\frac{1}{2}(\delta \boldsymbol{x} \nabla)^{2}+\cdots\right\} V_{0}(\boldsymbol{x}) .
$$

If we average the last equation in space, we can eliminate so called the effective potential in the form

$$
V(\boldsymbol{x})=\left\{1+\frac{1}{6}(\delta \boldsymbol{x})_{T}^{2} \Delta+\cdots\right\} V_{0}(\boldsymbol{x})
$$

where $(\delta \boldsymbol{x})_{T}^{2}$ is the average value of the square coordinate shift caused by the thermal photon fluctuations. The potential shift follows from Equation (2):

$$
\delta V(\boldsymbol{x})=\frac{1}{6}(\delta \boldsymbol{x})_{T}^{2} \Delta V_{0}(\boldsymbol{x}) .
$$

The corresponding shift of the energy levels is given by the standard quantum mechanical formula [8]

$$
\delta E_{n}=\frac{1}{6}(\delta \boldsymbol{x})_{T}^{2}\left(\psi_{n} \Delta V_{0} \psi_{n}\right) .
$$

In case of the Coulomb potential, which is the case of the $\mathrm{H}$-atom, we have

$$
V_{0}=-\frac{e^{2}}{4 \pi|\boldsymbol{x}|}
$$

Then for the $\mathrm{H}$-atom we can write

$$
\delta E_{n}=(2 \pi / 3)(\delta \boldsymbol{x})_{T}^{2} \frac{e^{2}}{4 \pi}\left|\psi_{n}(0)\right|^{2},
$$

where we used the following equation for the Coulomb potential 


$$
\Delta \frac{1}{|x|}=-4 \pi \delta(x)
$$

Motion of an electron in electric field is evidently described by elementary equation

$$
\delta\left(\frac{\mathrm{d}^{2}}{\mathrm{~d} t^{2}}\right) \boldsymbol{x}=\frac{e}{m} \boldsymbol{E}_{T},
$$

which can be transformed by the Fourier transformation into the following equation

$$
\left|\delta \boldsymbol{x}_{T \omega}\right|^{2}=\frac{1}{2}\left(\frac{e^{2}}{m^{2} \omega^{4}}\right) \boldsymbol{E}_{T \omega}^{2},
$$

where the index $\omega$ concerns the Fourier component of above functions.

On the basis of the Bethe idea of the influence of vacuum fluctuations on the energy shift of electron [10], the following elementary relations were used by Welton [9] and Akhiezer [8] and Berestetzkii et al. [11]:

$$
\frac{1}{2} \boldsymbol{E}_{\omega}^{2}=h \omega / 2
$$

and in case of the thermal bath of the blackbody, the last equation is of the following form [12]:

$$
\boldsymbol{E}_{T \omega}^{2}=\rho(\omega)=\left(\frac{\hbar \omega^{3}}{\pi^{2} c^{3}}\right) \frac{1}{\mathrm{e}^{\frac{\hbar \omega}{k T}}-1},
$$

because the Planck law in (11) was written as

$$
\rho(\omega)=G(\omega)\langle E\rangle=\left(\frac{\hbar \omega^{3}}{\pi^{2} c^{3}}\right) \frac{1}{\mathrm{e}^{\frac{\hbar \omega}{k T}}-1},
$$

where the term

$$
\langle E\rangle=\frac{\hbar \omega}{\mathrm{e}^{\frac{\hbar \omega}{k T}}-1}
$$

is the average energy of photons in the blackbody and

$$
G(\omega)=\left(\frac{\omega^{2}}{\pi^{2} c^{3}}\right)
$$

is the number of electromagnetic modes in the interval $\omega, \omega+\mathrm{d} \omega$.

Then,

$$
\left(\delta \boldsymbol{x}_{T \omega}\right)^{2}=\frac{1}{2}\left(\frac{e^{2}}{m^{2} \omega^{4}}\right)\left(\frac{\hbar \omega^{3}}{\pi^{2} c^{3}}\right) \frac{1}{\mathrm{e}^{\frac{\hbar \omega}{k T}}-1},
$$

where $\left(\delta \boldsymbol{x}_{T \omega}\right)^{2}$ involves the number of frequencies in the interval $(\omega, \omega+\mathrm{d} \omega)$.

So, after some integration, we get

$$
\left(\delta \boldsymbol{x}_{T}\right)^{2}=\int_{\omega_{1}}^{\omega_{2}} \frac{1}{2}\left(\frac{e^{2}}{m^{2} \omega^{4}}\right)\left(\frac{\hbar \omega^{3}}{\pi^{2} c^{3}}\right) \frac{\mathrm{d} \omega}{\mathrm{e}^{\frac{\hbar \omega}{k T}}-1}=\frac{1}{2}\left(\frac{e^{2}}{m^{2}}\right)\left(\frac{\hbar}{\pi^{2} c^{3}}\right) F\left(\omega_{2}-\omega_{1}\right),
$$


where $F(\omega)$ is the primitive function of the omega-integral

$$
J=\left(\frac{1}{\omega}\right) \frac{1}{\mathrm{e}^{\frac{\hbar \omega}{k T}}-1},
$$

which cannot be calculated by the elementary integral methods and it is not involved in the tables of integrals.

Frequencies $\omega_{1}$ and $\omega_{2}$ will be determined with regard to the existence of the fluctuation field of thermal photons. It was determined in case of the Lamb shift [9] [10] by means of the physical analysis of the interaction of the Coulombic atom with the surrounding fluctuation field. We suppose here that the Bethe and Welton arguments are valid and so we take the frequencies in the Bethe-Welton form. In other words, electron cannot respond to the fluctuating field if the frequency which is much less than the atom binding energy given by the Rydberg constant [13] $R_{\text {Ryxdberg }}=\frac{\alpha^{2} m c^{2}}{2}$. So, the lower frequency limit is

$$
\omega_{1}=R_{\text {Ryxdberg }} / \hbar=\frac{\alpha^{2} m c^{2}}{2 \hbar}
$$

where $\alpha \approx 1 / 137$ is so called the fine structure constant.

The specific form of the second frequency follows from the elementary argument, that we expect the effective cutoff, since we must neglect the relativistic effect in our nonrelativistic theory. So, we write

$$
\omega_{2}=\frac{m c^{2}}{\hbar}
$$

If we take the thermal function of the form of the geometric series

$$
\begin{aligned}
& \frac{1}{\mathrm{e}^{\frac{\hbar \omega}{k T}}-1}=q\left(1+q^{2}+q^{3}+\cdots\right) ; q=\mathrm{e}^{-\frac{\hbar \omega}{k T}}, \\
& \int_{\omega_{1}}^{\omega} q\left(1+q^{2}+q^{3}+\cdots\right) \frac{\mathrm{d} \omega}{\omega}=\ln |\omega|+\cdots+\sum_{k=1}^{\infty} \frac{\left(-\frac{\hbar \omega}{k T}\right)}{k ! k}+\cdots ;
\end{aligned}
$$

and the first thermal contribution is thermal contribution

$$
\text { thermal contribution }=\left(\ln \frac{\omega_{2}}{\omega_{1}}-\frac{\hbar}{k T}\left(\omega_{2}-\omega_{1}\right)\right) .
$$

Then, with Equation (6)

$$
\delta E_{n}=\frac{2 \pi}{3}\left(\frac{e^{2}}{m^{2}}\right)\left(\frac{\hbar}{\pi^{2} c^{3}}\right)\left(\ln \frac{\omega_{2}}{\omega_{1}}-\frac{\hbar}{k T}\left(\omega_{2}-\omega_{1}\right)\right)\left|\psi_{n}(0)\right|^{2},
$$

where [14]

$$
\left|\psi_{n}(0)\right|=\frac{1}{\pi n^{2} a_{0}^{2}}
$$

with 


$$
a_{0}=\frac{\hbar^{2}}{m e^{2}}
$$

Let us only remark that the numerical form of Equation (23) has deep experimental astrophysical meaning.

In article by author [15], which is the continuation of author articles on the finitetemperature Čerenkov radiation and gravitational Čerenkov radiation [16] [17], the temperature Green function in the framework of the Schwinger source theory was derived in order to determine the Coulomb and Yukawa potentials at finite-temperature using the Green functions of a photon with and without radiative corrections, and then by considering the processes expressed by the Feynman diagrams.

The determination of potential at finite temperature is one of the problems which form the basic ingredients of the quantum field theory (QFT) at finite temperature. This theory was formulated some years ago by Dolan and Jackiw [18], Weinberg [19] and Bernard [20] in 1974 and some of the first applications of this theory were the calculations of the temperature behavior of the effective potential in the Higgs sector of the standard model.

Information on the systematic examination of the finite temperature effects in quantum electrodynamics (QED) at one-loop order was given by Donoghue, Holstein and Robinett [21]. Partovi [22] in 1994 discussed the QED corrections to Planck's radiation law and photon thermodynamics,

A similar discussion of QED was published by Johansson, Peressutti and Skagerstam [23] and Cox et al. in 1984 [24].

Serge Haroche [25] and his research group in the Paris microwave laboratory used a small cavity for the long life-time of photon quantum experiments performed with the Rydberg atoms. We considered here the thermal gas corresponding to the GibbonsHawking theory of space-time (at temperature T) as the preamble for new experiments for the determination of the energy shift of $\mathrm{H}$-atom electrons interacting with the Gibbons-Hawking thermal gas. It is not excluded, that the observations performed by the well educated astro-experts will be the Nobelian ones.

\section{References}

[1] Corda, Ch. (2015) Precise Model of Hawking Radiation from the Tunneling Mechanism. Classical and Quantum Gravity, 32, Article ID: 195007. http://dx.doi.org/10.1088/0264-9381/32/19/195007

[2] Corda, Ch. (2015) Quasi-Normal Modes: The "Electrons" of Blak Holes as "Gravitational Atoms"? Implications for the Black Hole Information Puzzle. Advances in High Energy Physics, 2015, Article ID: 867601. http://dx.doi.org/10.1155/2015/867601

[3] Corda, Ch. (2015) Time Dependent Schrödinger Equation for Black Hole Evaporation: No Information Loss. Annals of Physics, 353, 71. http://dx.doi.org/10.1016/j.aop.2014.11.002

[4] Planck, M. (1900) Zur Theorie des Gesetzes der Energieverteilung im Normalspektrum. Verhandlungen der Deutschen Physikalischen Gesellschaft, 2, 237.

[5] Planck, M. (1901) Ueber das Gesetz der Energieverteilung im Normalspectrum. Annals of Physics, 4, 553. http://dx.doi.org/10.1002/andp.19013090310 
[6] Schöpf, H.-G. (1978) Theorie der Wärmestrahlung in historisch-kritischer Darstellung. Alademie/Verlag, Berlin.

[7] Einstein, A. (1917) Zur Quantentheorie der Strahlung. Physikalische Zeitschrift, 18, 121.

[8] Akhiezer, A.I. and Berestetzkii, V.B. (1953) Quantum Electrodynamics. GITTL, Moscow.

[9] Welton, Th. (1948) Some Observable Effects of the Quantum-Mechanical Fluctuations of the Electromagnetic Field. Physical Review, 74, 1157. http://dx.doi.org/10.1103/PhysRev.74.1157

[10] Bethe, H.A. (1947) The Electromagnetic Shift of Energy Levels. Physical Review, 72, 339. http://dx.doi.org/10.1103/PhysRev.72.339

[11] Berestetzkii, V.B., Lifshitz, E.M. and Pitaevskii, L.P. (1999) Quantum Electrodynamics. Butterworth-Heinemann, Oxford.

[12] Isihara, A. (1971) Statistical Mechanics. Academic Press, Pittsburgh.

[13] Rohlf, J.W. (1994) Modern Physics from $\alpha$ to $Z Z$. John Wiley \& Sons Ltd., Hoboken.

[14] Sokolov, A.A., Loskutov, Y.M. and Ternov, I.M. (1962) Quantum Mechanics. State Pedagogical Edition, Moscow. (In Russian)

[15] Pardy, M. (1994) The Two-Body Potential at Finite Temperature. CERN.TH.7397/94.

[16] Pardy, M. (1989) Finite-Temperature Čerenkov Radiation. Physics Letters A, 134, 357-359. http://dx.doi.org/10.1016/0375-9601(89)90734-2

[17] Pardy, M. (1989) Finite-Temperature Gravitational Čerenkov Radiation. International Journal of Theoretical Physics, 34, 951-959. http://dx.doi.org/10.1007/BF00674453

[18] Dolan, L. and Jackiw, R. (1974) Symmetry Behavior at Finite Temperature. Physical Review D, 9, 3320-3341. http://dx.doi.org/10.1103/PhysRevD.9.3320

[19] Weinberg, S. (1974) Gauge and Global Symmetries at High Temperature. Physical Review D, 9, 3357-3378. http://dx.doi.org/10.1103/PhysRevD.9.3357

[20] Bernard. C.W. (1974) Feynman Rules for Gauge Theories at Finite Temperature. Physical Review D, 9, 3312-3320. http://dx.doi.org/10.1103/PhysRevD.9.3312

[21] Donoghue, J.F., Holstein, B.R. and Robinett, R.W. (1985) Quantum Electrodynamics at Finite Temperature. Annals of Physics, 164, 233-276. http://dx.doi.org/10.1016/0003-4916(85)90016-8

[22] Partovi, H.M. (1994) QED Corrections to Plancks Radiation Law and Photon Thermodynamics. Physical Review D, 50, 1118-1124. http://dx.doi.org/10.1103/PhysRevD.50.1118

[23] Johansson, A.E., Peressutti, G. and Skagerstam, B.S. (1986) Quantum Field Theory at Finite Temperature: Renormalization and Radiative Corrections. Nuclear Physics B, 278, 324-342. http://dx.doi.org/10.1016/0550-3213(86)90216-6

[24] Cox, P.H., Hellman, W.S. and Yildiz, A. (1984) Finite Temperature Corrections to Field Theory: Electron Mass, Magnetic Moment, and Vacuum Energy. Annals of Physics, 154, 211-228. http://dx.doi.org/10.1016/0003-4916(84)90143-X

[25] Haroche, S. (2012) The Secrets of My Prizewinning Research. Nature, 490, 311. http://dx.doi.org/10.1038/490311a 
Submit or recommend next manuscript to SCIRP and we will provide best service for you:

Accepting pre-submission inquiries through Email, Facebook, LinkedIn, Twitter, etc. A wide selection of journals (inclusive of 9 subjects, more than 200 journals)

Providing 24-hour high-quality service

User-friendly online submission system

Fair and swift peer-review system

Efficient typesetting and proofreading procedure

Display of the result of downloads and visits, as well as the number of cited articles

Maximum dissemination of your research work

Submit your manuscript at: http://papersubmission.scirp.org/ 